\title{
Entropic bond descriptors from separated output-reduced communication channels in atomic orbital resolution
}

\author{
Dariusz Szczepanik • Janusz Mrozek
}

Received: 19 February 2010 / Accepted: 14 October 2010 / Published online: 29 October 2010

C The Author(s) 2010. This article is published with open access at Springerlink.com

\begin{abstract}
Communication Theory of Chemical Bond (CTCB) in atomic orbital resolution is used to define entropic bond orders of diatomic molecular fragments. Partial communication channels for separated information flows from atomic centers and two alternative output-reducion schemes with their entropic descriptors are proposed. Also two types of information that can be transmitted through communication system are identified: information about molecular electron occupations and information about bonding shares of atomic orbitals. The former is used to evaluate an average number of electrons engaged in bond forming process while the latter provides information about electron localization in chemical bond. Calculated entropic bond orders and their IT-covalency and IT-ionicity components are in good agreement with both chemical intuition and MO theory predictions.
\end{abstract}

Keywords Bond order - Communication channel · Information scattering · Covalency $\cdot$ Ionicity

\section{Introduction}

Communication Theory of Chemical Bond (CTCB) [1,2] has recently been formulated by Nalewajski as a part of work on using Information Theory (IT) [3] in probing molecular electronic structure. This IT-approach to chemical bond is based on the Shannon theory of communication [3] and provides tools for describing bonding patterns in both the ground [4] and exited electron configurations [5].

\footnotetext{
D. Szczepanik · J. Mrozek $(\varangle)$

Department of Computational Methods in Chemistry, Jagiellonian University,

R. Ingardena St. 3, 30-060 Cracow, Poland

e-mail: mrozek@chemia.uj.edu.pl
} 
The key concept of Orbital Communication Theory (OCT) [6] is the molecular information system in atomic orbital (AO) resolution. The input and output signal is defined through a set of atomic orbitals, $\chi=\left\{\chi_{i}\right\}$, and vectors of input and output probabilities of finding an electron on particular $\mathrm{AO}, \mathbf{p}=\left\{p_{i}\right\}$ and $\mathbf{q}=\left\{q_{i}\right\}$, respectively. The communication channel in the OCT is uniquely determined by conditional probability matrix, $\mathbf{P}(\chi \mid \chi)=\left\{P\left(\chi_{j} \mid \chi_{i}\right)\right\}$, where element $P\left(\chi_{j} \mid \chi_{i}\right)$ denotes probability of finding on $\chi_{j}$ (output) electron derived from $\chi_{i}$ (input).

In the OCT the information flow through a communication system is attributed to changes in electron occupations of particular atomic orbitals in the molecule. Information about electrons is scattered in the communication channel, and the noise in the OCT is related to delocalization of electrons. The conditional entropy, $S(\chi \mid \chi)$, measures the amount of such scattering, thus it is treated as a IT-covalency index of molecular system and the mutual information, $I(\chi: \chi)$, is measuring the amount of information which passes through the communication channel, thus it is specifying to what a degree electrons are attributed to particular atoms. Therefore, one may assume that it reflects the IT-ionicity of the molecular communication system. In OCT the overall IT-bond order is defined by the simple sum of conditional entropy and mutual information, $N(\chi ; \chi)=S(\chi \mid \chi)+I(\chi: \chi)$.

It has been shown [7] that additive decomposition of the global entropic quantities allows one to calculate information indices of chemical bonds on particular molecular fragment. Such decomposition also gives rise to division of the entropy descriptors into the corresponding internal and external contributions. The former measures an average amount of information dissipated between AOs centered on the same atom (fragment) whilst the latter measures an average amount of information dissipated between AOs originated from two (or more) different atoms (fragments). It has been demonstrated using simple atomic orbital models that entropic indices of diatomic fragments in the molecule are quite different from those generated by the related counterparts from Molecular Orbital (MO) theory. Proposed IT-descriptors emphasize the equilibrium of the bonded subsystems reflecting the molecular electronic ground state as a collection of the mutually open (bonded, connected) subsystems. The corresponding approach to the renormalized channels of the separate (externally closed, disconnected) molecular subsystems has also been explored [8].

An alternative set of internal and external information-theoretic quantities has been defined using the appropriate reduction of the molecular channel, by combining the fragment constituent atoms into condensed units [9]. The output reduction scheme determines the molecular parts, between which the effective external communications (bonds) are established, while the input reduction defines the communication sources (origins). For a given output reduction, a relatively weak dependence of the external bond indices on the assumed input reduction has been observed.

It has recently been shown [10] that the appropriate output reduction scheme might be essential in order to extract from molecular communication channel this part of information which correctly describes electron scattering during bond formation process between diatomic fragments in molecule. It is the main goal of this work to investigate closer newly proposed output reduction scheme for partial molecular channels (mutually open diatomic subsystem). Also some numerical results for both model and real molecular systems will be presented and briefly discussed. 


\section{Molecular channels in atomic orbital resolution}

Let as assume, for simplicity, the closed-shell molecular system with $N$ electrons doubly occupying the $n$ lowest (ground state), orthonormal MOs, $\psi=\left\{\psi_{1}, \psi_{2}, \ldots, \psi_{n}\right\}$, generated as linear combinations of the basis functions $\chi$, representing Löwdin's symmetrically Orthogonalized Atomic Orbitals (OAO), $\psi=\chi \mathbf{C}$, where the rectangular matrix $\mathbf{C}=\langle\chi \mid \psi\rangle=\left\{C_{i, s}\right\}$ groups the relevant LCAO MO expansion coefficients.

A set of such MOs uniquely defines the system electron density:

$$
\rho(\mathbf{r})=2 \boldsymbol{\psi}(\mathbf{r}) \boldsymbol{\psi}^{\dagger}(\mathbf{r})=\chi(\mathbf{r})\left[2 \mathbf{C} \mathbf{C}^{\dagger}\right] \chi^{\dagger}(\mathbf{r})=\chi(\mathbf{r}) \gamma \chi^{\dagger}(\mathbf{r}),
$$

where $\gamma$ is the so called Charge-and-Bond-Order (CBO) matrix satisfying idempotency relation appropriate for closed-shell systems, $(\gamma)^{2}=2 \gamma$. Diagonal elements of CBO matrix measure the effective electron occupations of atomic orbitals, $N_{i}=$ $N\left(\chi_{i}\right)=\gamma_{i, i}$, and in OCT they are used to define molecular (occupational) inputprobability vector, $\mathbf{p}$, :

$$
\mathbf{p}=\left\{p_{i} \equiv p\left(\chi_{i}\right)=\frac{N_{i}}{N}=\frac{\gamma_{i, i}}{N}\right\}
$$

where $N$ denotes total number of electrons in molecule as a whole, $N=\sum_{i} N_{i}$.

Communication network is determined by the conditional probabilities of the output AO-events, given the input AO-events, $\left\{P\left(\chi_{j} \mid \chi_{i}\right)\right\}$. It was shown $[6,10]$ using the generalized superposition principle of quantum mechanics, that for closed-shell molecular systems conditional probability matrix can be defined using the squares of corresponding elements of $\mathrm{CBO}$ matrix and its idempotency relation:

$$
\mathbf{P}(\chi \mid \chi)=\left\{P\left(\chi_{j} \mid \chi_{i}\right)=\mathcal{N}_{i}\left|\left\langle\chi_{i}\left|\hat{P}_{\psi}^{o}\right| \chi_{j}\right\rangle\right|^{2}=\frac{\gamma_{i, j} \gamma_{j, i}}{2 \gamma_{i, i}}\right\} .
$$

The off-diagonal probabilities $\left\{P\left(\chi_{j} \mid \chi_{i}\right), i \neq j\right\}$ are thus proportional to the corresponding AO's contributions $w_{i, j}=\gamma_{i, j}^{2}$ to the Wiberg covalency index [11] between two atoms, $\mathrm{A}$ and $\mathrm{B}, W_{A-B}=\sum_{i \in A} \sum_{i \in B} w_{i, j}$.

Molecular output-probability vector, $\mathbf{q}$, is determined by corresponding occupational input-probability vector (2) and conditional probability matrix in orbital resolution (3):

$$
\mathbf{q}=\mathbf{p P}(\chi \mid \chi)=\left\{q_{j}=\sum_{i} p_{i} P\left(\chi_{j} \mid \chi_{i}\right)=\sum_{i} P\left(\chi_{i} \wedge \chi_{j}\right)=p_{j}\right\}=\mathbf{p},
$$

where $P\left(\chi_{i} \wedge \chi_{j}\right)$ is the joint probability of simultaneously observing two AO-events and satisfies appropriate normalizations conditions, $\sum_{i} P\left(\chi_{i} \wedge \chi_{j}\right)=p_{j}$, $\sum_{j} P\left(\chi_{i} \wedge \chi_{j}\right)=p_{i}$, and $\sum_{i} \sum_{j} P\left(\chi_{i} \wedge \chi_{j}\right)=1$. As we can see, in a simple nonreduced communication systems molecular input generates the same distribution in the 
output of the molecular channel. Thus $\mathbf{p}$ might be regarded as the stationary probability vector for the molecular ground state.

Amount of information included in molecular input probability distribution can be simply measured (in bits) using Shannons entropy:

$$
H[\mathbf{p}]=\sum_{i} p_{i} \log _{2} p_{i}
$$

The fraction of the information content in $\mathbf{p}$, which has not been scattered in the molecular communication system, is given by mutual information:

$$
I(\chi: \chi)=\sum_{i} p_{i} \sum_{j} P\left(\chi_{j} \mid \chi_{i}\right) \log _{2} \frac{P\left(\chi_{j} \mid \chi_{i}\right)}{p_{j}} .
$$

The maximum information that can be transmitted through the communication channel is called the channel capacity,

$$
C(\chi: \chi)=\sup _{\mathbf{p}} I(\chi: \chi)
$$

Finally, amount of information that has been dissipated as a noise in the molecular information system is measured by the conditional entropy:

$$
S(\chi \mid \chi)=\sum_{i} p_{i} \sum_{j} P\left(\chi_{j} \mid \chi_{i}\right) \log _{2} P\left(\chi_{j} \mid \chi_{i}\right)
$$

Conditional entropy, $S(\chi \mid \chi)$, and mutual information, $I(\chi: \chi)$, play essential role within Orbital Communication Theory. They are used as IT-covalency and IT-ionicity descriptors, respectively. Overall IT-bond order in such approach is proposed as simple sum

$$
N(\chi ; \chi)=S(\chi \mid \chi)+I(\chi: \chi)=\sum_{i} p_{i} \log _{2} p_{i}=H[\mathbf{p}]
$$

It has been demonstrated many times $[1,2]$ that such entropy indices constitute useful and effective tools being — in the case of simple orbital models_-generally in good agreement with both MO's indices and chemical intuition.

Let us briefly discuss the simplest 2-AO model of hydrogen molecule, in which we observe interaction between the two orthogonal atomic orbitals, $\chi_{A}$ and $\chi_{B}$, originating from atoms $\mathrm{A}$ and $\mathrm{B}$, respectively, which gives rise to the two orthonormal combinations: bonding $\mathrm{MO}, \psi_{b}$, and antibonding $\mathrm{MO}, \psi_{a}$. In such 2-AO model conditional entropy is equivalent to so called binary entropy function [1,12], $H_{b}(p)=-p \log p-(1-p) \log (1-p)$, and the maximum value it reaches is $1 \mathrm{bit}$. For uniform input probability distribution, $\mathbf{p}=\left\{\frac{1}{2}, \frac{1}{2}\right\}$, representing a typical covalent bond (each atom provides only one electron), overall IT-bond order index gains a maximum, constant value, $N_{b}=1$, regardless of MO's polarization; conditional entropy 
and mutual information amount to 1 and 0 , respectively, what indicates a purely covalent chemical bond. When we take input probability distribution $\mathbf{p}=\{0,1\}$ and fully polarized bond, we obtain $S_{b}=0, I_{b}=0$, and overall index $N_{b}=0$ which represents no interaction between doubly occupied one orbital with unoccupied another.

An interesting case of 2-AO communication system with $\mathbf{p}=\{0,1\}$ constitutes channel with non-polarized MO. For this theoretical model of coordination bond we obtain: $S_{b}=1, I_{b}=-1$, and overall bond order $N_{b}=0$, which fully disagree both with chemical intuition and standard MO theory. Alternative definition of IT-bond multiplicity based on the channel capacity (7) instead of the mutual information (6) will be discussed and examined further in next sections, but now we can simply compare binary channel capacity and binary mutual information results for 2-AO model of hydrogen molecule: $S_{b}=1, C_{b}=0$, and overall bond order $\tilde{N}_{b}=S_{b}+C_{b}=1$. Therefore, using $C_{b}$ as an index of IT-ionicity dramatically improves values of overall bond multiplicity.

Orbital Communication Theory of Chemical Bond predicts overall IT-bond orders properly describing molecular reality of such $\pi$-conjugated systems as butadiene and benzene $[4,5]$. In butadiene we observe 2-bits (IT-double) overall bond index of mainly entropy-covalent origin whereas in benzene the overall IT-multiplicity equals 2.59 thus falling short of the 3-bits measure predicted for three separated $\pi$ bonds in cycloheksatriene. This numerical values qualitatively agree with the intuitive (chemical) $\pi$-bond multiplicities for these systems of two conjugated bonds in butadiene and approximately three delocalized bonds in benzene.

\section{Diatomic IT-indices of molecular fragments}

In order to extract entropy indices between particular two atoms in a molecule one can decompose information system into so called partial row channels and then sum up all contributions from the specified fragment. If we define, for example, a diatomic fragment $\mathbf{L}=\left\{\chi_{A}, \chi_{B}\right\}$ then the conditional entropy decomposition gives:

$$
S(\chi \mid \mathbf{L})=S^{i n t}(\mathbf{L} \mid \mathbf{L})+S^{e x t}(\chi \notin \mathbf{L} \mid \mathbf{L})=\sum_{i \in L} p_{i} \sum_{j} P\left(\chi_{j} \mid \chi_{i}\right) \log _{2} P\left(\chi_{j} \mid \chi_{i}\right)
$$

Internal conditional entropy, $S^{\text {int }}(\mathbf{L} \mid \mathbf{L})$, measures averaged amount of information scattered between two atoms A and B, giving rise to diatomic IT-covalency index. The same way one can decompose mutual information and overall IT-multiplicity of diatomic fragment $\mathrm{L}$ into internal and external contributions. It has been shown [7] that such internal descriptors of molecular fragments emphasize the information equilibrium of the almost purely covalent bonded subsystems. It is manifested by a remarkable equalization of various diatomic indices, irrespectively of the fragment position in a molecule.

Alternatively, so called reduced (condensed) communication channels have been engaged to probe chemical bond within IT. The reduction can be carried by combining the fragment constituent atoms into condensed units. It has been widely argued $[1,9]$ that the output reduction scheme determines the molecular parts, between which the 
effective external communications (bonds) are established, while the input reduction defines the communication sources (origins).

The idea of partial communication channels supported by appropriate output reduction will be used now to present an alternative scheme for extracting diatomic bond orders within OCT. Hitherto, an effect of electrons flow (during bond formation process) from atoms into the space between them was linked to scattering of information about electron occupations of these atoms in communication channel. The key concept of such approach lies in the simultaneous scattering of information about electrons from each constituent atom of diatomic fragment which results from taking into account both these atoms in defining input probability vector, p. But an electron scattering in molecular communication channel can also be probed by building two separate atomic row channels, which depict directed information flow from atom A to B and vice versa. In such individual molecular sub-channels the source contains only atomic orbitals originated from specified atom in diatomic fragment of interest.

During bond formation process certain amount of electrons from atom A is being scattered "in direction" of atom B and simultaneously certain (generally not the same) amount of electrons from atom B is being scattered "in direction" of atom A. One could regard these partial processes as independent and link them directly to appropriate row channels ( $A \rightarrow B$ and $B \rightarrow A$ electron scattering). The essence of the newly proposed channel's reduction scheme is that in a single (partial) row communication we are interested in uncertainty of finding an electron originated from particular $i$ th atomic orbital of atom $\mathrm{A}, \chi_{i} \in \chi_{A}$, on whichever of AOs of the same atom, $\chi_{A}=\left\{\chi_{i}: i \in A\right\}$, or on whichever of the remaining atomic orbitals, $\chi_{A^{C}}=\left\{\chi_{i}: i \notin A\right\}$, where $A^{C}$ denotes a complement of $\mathrm{A}$ in $\mathrm{M}$ (the whole molecule). To do that, first we have to combine appropriate columns of conditional probability matrix (3) into two condensed units (columns), $\mathbf{P}\left(A \mid \chi_{A}\right)$ and $\mathbf{P}\left(A^{C} \mid \chi_{A}\right)$. Such condensation of individual column communications for both scattering directions, $A \rightarrow B$ and $B \rightarrow A$, gives the following output-reduced conditional probability (column) matrices:

$$
\begin{gathered}
\mathbf{P}\left(X \mid \chi_{X}\right)=\sum_{j \in X} \mathbf{P}\left(\chi_{j} \mid \chi_{X}\right)=\left\{P\left(X \mid \chi_{i}\right) \equiv \sum_{j \in X} \frac{\gamma_{i, j} \gamma_{i, j}}{2 \gamma_{i, i}}: i \in X\right\}, \quad X=A, B \\
\mathbf{P}\left(X^{C} \mid \chi_{X}\right)=\left\{P\left(X^{C} \mid \chi_{i}\right) \equiv 1-P\left(X \mid \chi_{i}\right): i \in X\right\}, \quad X=A, B .
\end{gathered}
$$

Therefore, within this output-reduction scenario, for $i$ th row of condensed conditional probability column matrix of $X \rightarrow X^{C}$ (rather than $A \rightarrow B$ or $B \rightarrow A$ ) electron scattering we are interested in the probability of finding an electron derived from $\chi_{i}$ on atom $\mathrm{X}, P\left(X \mid \chi_{i}\right)$, or any other atom in molecule, $P\left(X^{C} \mid \chi_{i}\right)$.

Occupational input probabilities related to atoms $\mathrm{A}$ and $\mathrm{B}$ are now defined as renormalized molecular input probabilities from Eq. 2: 


$$
\mathbf{P}_{X}=\left\{P_{i \mid X}=\frac{N_{i}}{N_{X}}=\frac{\gamma_{i, i}}{\sum_{j \in X} \gamma_{j, j}}: i \in X\right\}, \quad X=A, B,
$$

with normalization condition $\sum_{i \in X} P_{i \mid X}=1$, where $P_{i \mid X}$ stands for the conditional probability of finding an electron of atom X (A or B) on its constituent orbital $\chi_{i}$.

Condensation of conditional probability matrix's columns also implies the following output-reduced probabilities of both $A \rightarrow A^{C}$ and $B \rightarrow B^{C}$ channels:

$$
\mathbf{Q}_{X}=\left\{Q_{X}(X)=\sum_{i \in X} P_{i \mid X} P\left(X \mid \chi_{i}\right), Q_{X}\left(X^{C}\right)=1-Q_{X}(X)\right\}, \quad X=A, B
$$

We have to emphasize here that, according to Eq. 4 and output-reduced conditional probability matrix definition (11), vector $\mathbf{P}_{X}$ cannot be further regarded as the stationary probability vector for the molecular ground state. Moreover, $\mathbf{P}_{X}$ and $\mathbf{Q}_{X}$ are completely different with respect to number of elements: $\mathbf{Q}_{X}$ has only 2 elements (condensed fragment probabilities) whilst number of elements in $\mathbf{P}_{X}$ depends on basis set. Figure 1 shows exemplary output-reduced communication channels $C \rightarrow C^{C}$ and $H \rightarrow H^{C}$ of chemical bond $\mathrm{C}-\mathrm{H}$ in typical hydrocarbons using minimal AO's basis set.
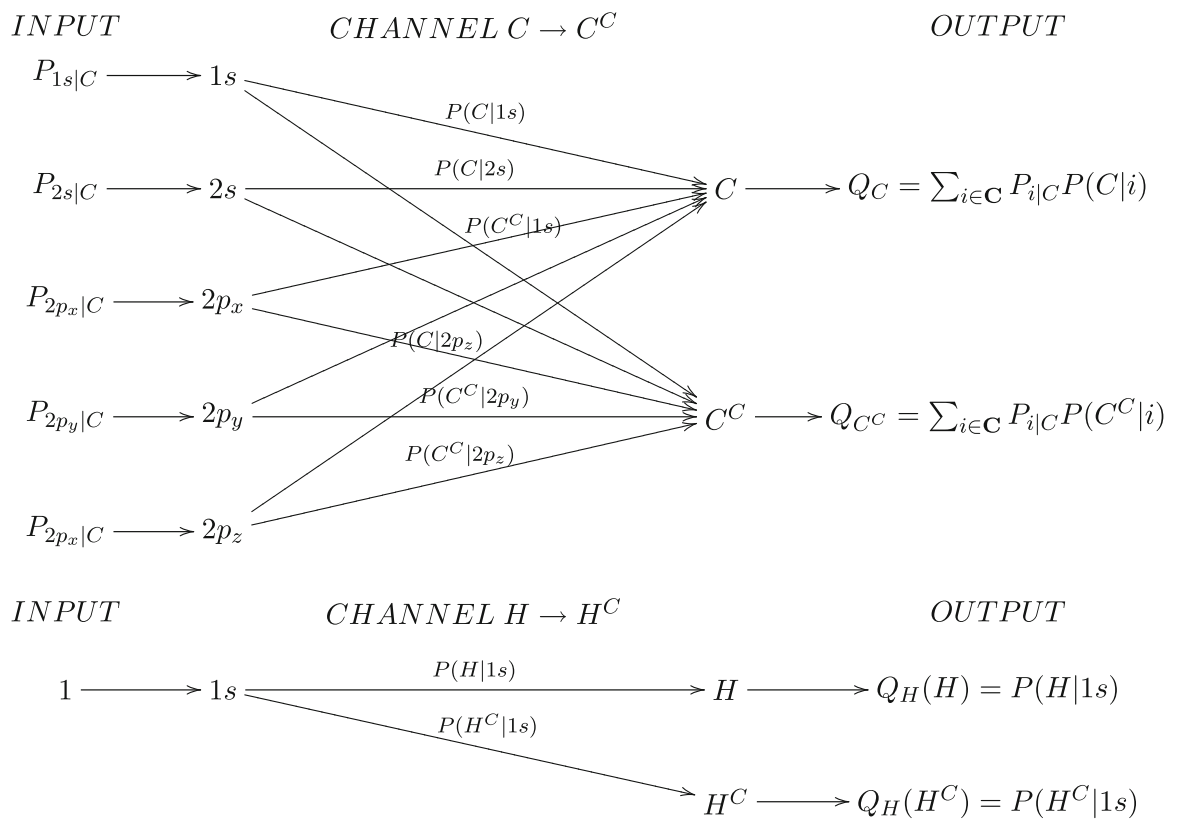

Fig. 1 Separated communication channels $C \rightarrow C^{C}$ and $H \rightarrow H^{C}$ of chemical bond C-H in typical hydrocarbons with minimal AO' basis set adopted 
Therefore, according to Eqs. 8, 11 and 12, the reduced conditional entropy of each partial communication channel is given by

$$
S_{X}=-\sum_{i \in X} P_{i \mid X}\left[P\left(X \mid \chi_{i}\right) \log _{2} P\left(X \mid \chi_{i}\right)+P\left(X^{C} \mid \chi_{i}\right) \log _{2} P\left(X^{C} \mid \chi_{i}\right)\right], \quad X=A, B
$$

and the reduced mutual information, according to Eqs. 6, 11, 12 and 13, reads

$$
I_{X}=-\sum_{i \in X} P_{i \mid X}\left[P\left(X \mid \chi_{i}\right) \log _{2} \frac{P\left(X \mid \chi_{i}\right)}{Q_{X}(X)}+P\left(X^{C} \mid \chi_{i}\right) \log _{2} \frac{P\left(X^{C} \mid \chi_{i}\right)}{Q_{X}\left(X^{C}\right)}\right], X=A, B
$$

The maximal value that these entropic entities gain is 1 bit. Moreover, maximization of reduced mutual information (15) with respect to the output probability vector (which for particular communication channel is uniquely determined by input probability distribution) gives the reduced information capacity:

$$
C_{X}=\max _{\mathbf{P}_{X}} I_{X}=\max _{\mathbf{P}_{X}} H\left(\mathbf{P}_{X}\right)-S_{X}=1-S_{X}, \quad X=A, B
$$

The sum of channel information capacity (amount of information that can be transmitted through the channel) and conditional entropy (amount of information that is dissipated; changed into noise) is constant and independent on input/output probability distribution. It depends only on a number of columns in conditional probability matrix [12], or in other words a number of events (orbitals) on communication channel output, $r_{\text {out }}$ :

$$
C_{X}+S_{X}=\log _{2} r_{\text {out }}=\log _{2} 2=1 \text { bit }, \quad X=A, B
$$

Using reduced conditional entropy (14) and information capacity (16) (instead of mutual information (15) we can straightforwardly express amount of information about electron allocations on atom $\mathrm{X}$ lost during bond forming process by a number of electrons intead of number of bits:

$$
V_{X}^{i n f}=\frac{S_{X}}{C_{X}+S_{X}} N_{A}=\frac{S_{X}}{1 \text { bit }}\left(\sum_{i \in X} \gamma_{i, i}\right) \geq 0, \quad X=A, B
$$

Since $V_{X}^{\text {inf }}$ is related to the overall number of electrons that are being scattered from particular atom (channel $X \rightarrow X^{C}$ ) one can interpret it as the IT-atomic valency descriptors. In molecules larger than diatomics only a fraction of electrons dissipated from atom $\mathrm{A}$ is engaged in bonding with atom $\mathrm{B}(A \rightarrow B)$, and vice versa $(B \rightarrow A)$. Thus, we have to multiply entities from preceding equation $\left(V_{A}\right.$ and $\left.V_{B}\right)$ by the 
condenced bonding shares, $w_{A \rightarrow B}$ and $w_{B \rightarrow A}$, defined by

$$
\begin{array}{r}
w_{A \rightarrow B}=\frac{\sum_{i \in A} \sum_{j \in B} P_{i \mid A} P\left(\chi_{j} \mid \chi_{i}\right)}{\sum_{i \in A} P_{i \mid A} P\left(A^{C} \mid \chi_{i}\right)}=\frac{\sum_{i \in A} \sum_{j \in B} \gamma_{i, j} \gamma_{j, i}}{\sum_{i \in A} \sum_{j \notin A} \gamma_{i, j} \gamma_{j, i}}, \\
w_{B \rightarrow A}=\frac{\sum_{i \in B} \sum_{j \in A} P_{i \mid B} P\left(\chi_{j} \mid \chi_{i}\right)}{\sum_{i \in B} P_{i \mid B} P\left(B^{C} \mid \chi_{i}\right)}=\frac{\sum_{i \in B} \sum_{j \in A} \gamma_{i, j} \gamma_{j, i}}{\sum_{i \in B} \sum_{j \notin B} \gamma_{i, j} \gamma_{j, i}},
\end{array}
$$

in order to determine $N_{A \rightarrow B}^{\text {inf }}$ and $N_{B \rightarrow A}^{\text {inf }}$ :

$$
\begin{aligned}
& N_{A \rightarrow B}^{i n f}=w_{A \rightarrow B} V_{A}^{i n f}=\frac{w_{A \rightarrow B} S_{A}}{1 \mathrm{bit}} N_{A}, \\
& N_{B \rightarrow A}^{i n f}=w_{B \rightarrow A} V_{B}^{i n f}=\frac{w_{B \rightarrow A} S_{B}}{1 \mathrm{bit}} N_{B} .
\end{aligned}
$$

$N_{A \rightarrow B}^{i n f}\left(N_{A \rightarrow B}^{i n f}\right)$ denotes a number of electrons dissipated from atom A (B) "in direction" of atom B (A). Using entities from Eq. 20 we can define the IT-bond multiplicity as an average number of electrons from both atoms engaged in chemical bond,

$$
N_{A-B}^{i n f}=\frac{N_{A \rightarrow B}^{i n f}+N_{B \rightarrow A}^{i n f}}{2} .
$$

Furthermore, one can regard the difference between $N_{A \rightarrow B}^{i n f}$ and $N_{B \rightarrow A}^{\text {inf }}$ as a IT-descriptor of chemical bond's polarization (a measure of asymmetry of electron density distribution between $\mathrm{A}$ and $\mathrm{B}$ ),

$$
\Delta N_{A-B}^{i n f}=\left|N_{A \rightarrow B}^{i n f}-N_{B \rightarrow A}^{i n f}\right|
$$

This novel definition of IT-bond order has been used to evaluate multiplicities of carbon-carbon bonds in ethane, butadiene and benzene within Hückel approximation and results are presented in Table 1. For comparison also Wigerg's covalency indices from MO theory and internal parts of the reduced IT-bond descriptors of diatomic row-channels from the previous approach [9] are given.

A reference to Table 1 indicates, that IT-bond indices of separated partial communication channels, $A \rightarrow B$ and $B \rightarrow A$, with proposed output-reduction scheme predict purely covalent (non-polarized) $\pi$-type bonds in all three molecules, with full agreement with $N_{A-B}^{i n t}$ decomposition predictions (very small values of IT-ionicity, $I_{A-B}^{i n t}$ ) and chemical intuition. But in contrast to internal quantities newly proposed IT-multiplicities have strongly different values depending on atoms position in molecule. In butadiene $N_{A-B}^{i n f}$ predicts the strongest $\pi$-bond between carbon atoms $C_{1}-C_{2}$ and $C_{3}-C_{4}$ and very week interaction between carbon atoms $C_{2}-C_{3}$, while $N_{A-B}^{i n t}$ predicts nearly $\pi$-single bond $(0.89 \div 1.00)$ regardles of distance between atoms. Similarly, in benzene molecule $N_{A-B}^{i n f}$ allows one to distinguish strong interaction between carbon atoms in position orto from weak interaction between carbon atoms in position 
Table 1 Comparison of the newly proposed IT-bond indices from separated molecular channels to the previous [7], internal parts of reduced IT-bond descriptors of diatomic fragment row-channels, $N_{A-B}^{i n t}, S_{A-B}^{i n t}, I_{A-B}^{i n t}$ and Wiberg's covalency indices, $W_{A-B}$

\begin{tabular}{lllllllll}
\hline Index & Ethane & \multicolumn{2}{c}{ Butadiene } & \multicolumn{7}{c}{ Benzene } \\
& $C-C$ & $C_{1}-C_{2}$ & $C_{1}-C_{3}$ & $C_{1}-C_{4}$ & $C_{2}-C_{3}$ & $o-C-C$ & $m-C-C$ & $p-C-C$ \\
\hline$W_{A-B}$ & 1.000 & 0.800 & 0.000 & 0.200 & 0.200 & 0.440 & 0.000 & 0.110 \\
$N_{A-B}$ & 1.000 & 0.800 & 0.000 & 0.200 & 0.200 & 0.440 & 0.000 & 0.110 \\
$\Delta N_{A-B}$ & 0.000 & 0.000 & 0.000 & 0.000 & 0.000 & 0.000 & 0.000 & 0.000 \\
$N_{A-B}^{\text {int }}$ & 1.000 & 0.894 & 1.000 & 0.980 & 0.980 & 0.759 & 0.822 & 0.807 \\
$S_{A-B}^{\text {int }}$ & 1.000 & 0.896 & 0.960 & 0.952 & 0.952 & 0.750 & 0.798 & 0.786 \\
$I_{A-B}^{i \text { int }}$ & 0.000 & -0.002 & 0.040 & 0.028 & 0.028 & 0.009 & 0.024 & 0.021 \\
\hline
\end{tabular}

para. Moreover, it predicts no interaction between carbon atoms in position meta. In case of $N_{A-B}^{i n t}$ again we observe equalization of all chemical interactions between carbon atoms. Therefore, decomposition of molecular communication channel into two independent subchannels with condensed outputs remarkably improve the results. In addition, obtained values determine average numbers of electrons about which two interacting atoms lose information instead of numbers of information's bits, like in the previous approaches.

It follows directly from Table 1 that index $N_{A-B}^{\text {inf }}$ assumes values exactly equal to Wiberg's covalency index, $W_{A-B}$. In order to examine closer the proposed IT-descriptors of chemical bond we have calculated one-electron density matrices for several representative molecules using method DFT/B3LYP and 3-21G basis set. The results presented in Table 2 indicates that for several molecules $N_{A-B}^{\text {inf }}$ differs meaningfully from $W_{A-B}$, especially for highly polarized chemical bonds. In case of all organic molecules with covalent chemical bonds IT-multiplicities assume values comparable to Wiberg's indices. An interesting case is a homonuclear $F_{2}$ molecule. Calculated IT-multiplicity of F-F chemical bond is nearly half as high as expected which means that both fluorine atoms lose information about 1.5 electrons due to chemical interaction. It will be shown in the next section that a half of this "extra" electron results from influence of lone pairs centered on both atoms.

\section{IT-ionicity concept within OCT}

In the preceding section we have used diagonal elements of the first order density matrix to determine molecular (occupational) input probabilities (12) for two complementary partial communications, $A \rightarrow B$ and $B \rightarrow A$. The proposed bond polarity IT-measure (22) properly distinguishes between (fully) covalent and (highly) polarized chemical bonds. However, in case of lithium fluoride $\Delta N_{A-B}$ gains too high value wrongly suggesting transfer about 2.5 electrons from $\mathrm{Li}$ to $\mathrm{F}$. To improve the method we can involve so called bond-weighted channel concept [10]. The idea consists in using renormalized joint probabilities (4) instead of occupational probabilities (12) in 
Table 2 Comparison of newly proposed IT-bond indices with Wiberg covalency indices, $W_{A-B}$

\begin{tabular}{|c|c|c|c|c|c|c|c|c|}
\hline Molecule & Bond & $V_{A}^{i n f}$ & $N_{A \rightarrow B}^{i n f}$ & $V_{B}^{i n f}$ & $N_{B \rightarrow A}^{i n f}$ & $\Delta N_{A-B}^{i n f}$ & $N_{A-B}^{i n f}$ & $W_{A-B}$ \\
\hline Hydrogen & $\mathrm{H}-\mathrm{H}$ & 1.000 & 1.000 & 1.000 & 1.000 & 0.000 & 1.000 & 1.000 \\
\hline Fluorine & $\mathrm{F}-\mathrm{F}$ & 1.436 & 1.436 & 1.436 & 1.436 & 0.000 & 1.436 & 1.087 \\
\hline Hydrogen fluoride & $\mathrm{H}-\mathrm{F}$ & 0.670 & 0.670 & 1.749 & 1.749 & 1.079 & 1.209 & 0.946 \\
\hline Lithium fluoride & $\mathrm{Li}-\mathrm{F}$ & 0.506 & 0.506 & 3.188 & 3.188 & 2.682 & 1.847 & 1.318 \\
\hline Carbon monoxide & $\mathrm{CO}$ & 2.986 & 2.986 & 4.762 & 4.762 & 1.775 & 3.874 & 2.916 \\
\hline Carbon dioxide & $\mathrm{C}-\mathrm{O}$ & 3.846 & 1.923 & 4.626 & 3.819 & 1.896 & 2.871 & 2.213 \\
\hline Water & $\mathrm{O}-\mathrm{H}$ & 3.165 & 1.582 & 0.754 & 0.749 & 0.833 & 1.165 & 0.966 \\
\hline Ammonia & $\mathrm{N}-\mathrm{H}$ & 4.018 & 1.339 & 0.825 & 0.813 & 0.526 & 1.076 & 0.982 \\
\hline Methane & $\mathrm{C}-\mathrm{H}$ & 4.456 & 1.114 & 0.895 & 0.878 & 0.236 & 0.996 & 0.989 \\
\hline Ethane & $\mathrm{C}-\mathrm{C}$ & 4.358 & 1.180 & 4.358 & 1.180 & 0.000 & 1.180 & 1.092 \\
\hline Ethane & $\mathrm{C}-\mathrm{H}$ & 4.358 & 1.047 & 0.905 & 0.868 & 0.179 & 0.958 & 0.969 \\
\hline Ethylene & $\mathrm{C}-\mathrm{C}$ & 4.263 & 2.234 & 4.263 & 2.234 & 0.000 & 2.234 & 2.120 \\
\hline Ethylene & $\mathrm{C}-\mathrm{H}$ & 4.263 & 1.004 & 0.899 & 0.847 & 0.157 & 0.925 & 0.952 \\
\hline Acetylene & $\mathrm{C}-\mathrm{C}$ & 4.241 & 3.217 & 4.241 & 3.217 & 0.000 & 3.217 & 3.100 \\
\hline Acetylene & $\mathrm{C}-\mathrm{H}$ & 4.241 & 0.971 & 0.847 & 0.795 & 0.176 & 0.883 & 0.936 \\
\hline Butadiene & $\mathrm{C}_{1}-\mathrm{C}_{2}$ & 4.290 & 2.079 & 4.217 & 2.002 & 0.039 & 2.040 & 1.971 \\
\hline Butadiene & $\mathrm{C}_{1}-\mathrm{C}_{3}$ & 4.290 & 0.038 & 4.217 & 0.037 & 0.000 & 0.037 & 0.036 \\
\hline Butadiene & $\mathrm{C}_{1}-\mathrm{C}_{4}$ & 4.290 & 0.133 & 4.290 & 0.133 & 0.000 & 0.133 & 0.126 \\
\hline Butadiene & $\mathrm{C}_{2}-\mathrm{C}_{3}$ & 4.217 & 1.143 & 4.217 & 1.143 & 0.000 & 1.143 & 1.125 \\
\hline Benzene & $\mathrm{C}_{n}-\mathrm{C}_{n+1}$ & 4.216 & 1.500 & 4.216 & 1.500 & 0.000 & 1.500 & 1.475 \\
\hline Benzene & $\mathrm{C}_{n}-\mathrm{C}_{n+2}$ & 4.216 & 0.037 & 4.215 & 0.037 & 0.000 & 0.037 & 0.036 \\
\hline Benzene & $\mathrm{C}_{n}-\mathrm{C}_{n+3}$ & 4.216 & 0.125 & 4.216 & 0.125 & 0.000 & 0.125 & 0.123 \\
\hline
\end{tabular}

First order density matrix was generated using DFT/B3LYP method and 3-21G basis set

defining input probability vector. Therefore, in such communication channel information about bonding probabilities is propagated.

The bonding probability vector determining shares of each atomic orbital centered on atom $\mathrm{X}$ in bonding with the rest of atoms is given by

$$
\mathbf{P}_{\mathbf{X}, X^{C}}=\left\{P_{i, X^{C}}=\frac{P_{i \mid X} P\left(X^{C} \mid i\right)}{\sum_{i \in X} P_{i \mid X} P\left(X^{C} \mid i\right)}: i \in X\right\}, \quad X=A, B .
$$

Using joint probabilities $\left\{P_{i, X^{C}}\right\}$ instead of $\left\{P_{i \mid X}\right\}$ in Eqs. 14 gives rise to reduced conditional entropy of electrons originated from atom $\mathrm{X}$ and engaged in chemical bonds with other atoms

$$
\mathscr{S}_{X}=-\sum_{i \in X} P_{i, X^{C}}\left[P\left(X \mid \chi_{i}\right) \log _{2} P\left(X \mid \chi_{i}\right)+P\left(X^{C} \mid \chi_{i}\right) \log _{2} P\left(X^{C} \mid \chi_{i}\right)\right], \quad X=A, B .
$$


Thus, conditional entropy (24) allows one to evaluate degree of electron delocalization via chemical bond between atoms A and B. Moreover, according to Eq. 18 we can decompose IT-atomic valency index into two components: "locational" (ionic) and "delocational" (covalent):

$$
V_{X}^{\text {inf }}=V_{X}^{\text {del }}+V_{X}^{\text {loc }}=\left[\frac{\mathscr{S}_{X} S_{X}}{(1 \text { bit })^{2}} N_{X}\right]+\left[\frac{\left(1-\mathscr{S}_{X}\right) S_{X}}{(1 \mathrm{bit})^{2}} N_{X}\right], \quad X=A, B .
$$

These components determine number of electrons originated from atom $X \in(A, B)$ and well localized on atoms in molecule $\left(V_{X}^{\text {loc }}\right)$ or totally delocalized between atoms in molecule $\left(V_{X}^{\text {del }}\right)$.

Similarly, according to Eq. 20 and using previously defined condensed bonding shares (19) we can finally decompose entropic multiplicities $N_{A-B}^{\text {inf }}(21)$ into covalent $\left(N_{A-B}^{c o v}\right)$ and ionic component $\left(N_{A-B}^{i o n}\right)$ :

$$
\begin{aligned}
& N_{A-B}^{c o v}=\frac{1}{2}\left(w_{A \rightarrow B} V_{A}^{d e l}+w_{B \rightarrow A} V_{B}^{d e l}\right), \\
& N_{A-B}^{i o n}=\frac{1}{2}\left(w_{A \rightarrow B} V_{A}^{l o c}+w_{B \rightarrow A} V_{B}^{l o c}\right) .
\end{aligned}
$$

$N_{A-B}^{c o v}$ denotes an average amount of information lost by both bonded atoms (during bond A-B forming process) and expressed by a number of electrons or-in other words - number of electrons delocalized via chemical bond A-B. $N_{A-B}^{i o n}$ measures an average number of electrons well localized in chemical bond A-B, i.e., an average amount of information about electrons retained by both atoms $\mathrm{A}$ and $\mathrm{B}$.

Table 3 presents results of calculations of $V_{X}^{\text {del }}, V_{X}^{\text {loc }}, N_{A-B}^{i o n}, N_{A-B}^{c o v}$ and $W_{A-B}$ obtained for all molecules from Table 2. It follows from Table 3 that components $N_{A-B}^{c o v}$ and $N_{A-B}^{i o n}$ quite well reproduce chemical bond's character in molecules. IT-ionicity index assumes negligible values for homonuclear bonds $\mathrm{C}-\mathrm{C}$ as well as for $\mathrm{C}-\mathrm{H}$ bonds in all hydrocarbons. $N_{A-B}^{i o n}$ visibly increases in the following order: $\mathrm{C}-\mathrm{H}$, $\mathrm{N}-\mathrm{H}, \mathrm{O}-\mathrm{H}, \mathrm{F}-\mathrm{H}$ and in case of $\mathrm{LiF}$ molecule it clearly suggests transfer on average one electron between atoms $\mathrm{A}$ and $\mathrm{B}\left(N_{A-B}^{i o n}=1.00\right)$. IT-covalency index, on the other hand, for overwhelming majority of molecules assumes values comparable with Wiberg's covalency index which implies dominant role of entropic effects in bond forming process especially for weakly polarized and homonuclear chemical bonds. Difference between $N_{A-B}^{c o v}$ and $W_{A-B}$ for LiF molecule might result from relatively small AO's basis set adopted. We have to stress that in spite of $N_{A-B}^{i o n}$ value suggesting transfer exactly one electron from atom A to atom B the IT-covalency component, $N_{A-B}^{c o v}$, still reaches quite high value (but considerably lower than $W_{A-B}$ ). It seems understandable if we take under consideration the fact that purely ionic chemical bond would be related to a diagonal CBO matrix of non-interacting atoms. Therefore, an existence of chemical bonds is always connected to non-vanishing values of $W_{A-B} \mathrm{i}$ $N_{A-B}^{c o v}$. So, it is strongly recommended to analize IT-ionicity and IT-covalency indices independently: $N_{A-B}^{i o n}$ as a measure of electron transfer effect or-in other words-as 
Table 3 Comparison of the newly proposed IT-ionicity and IT-covalency descriptors of chemical bond with Wiberg covalency indices, $W_{A-B}$

\begin{tabular}{|c|c|c|c|c|c|c|c|c|}
\hline Molecule & Bond & $V_{A}^{d e l}$ & $V_{A}^{l o c}$ & $V_{B}^{d e l}$ & $V_{B}^{l o c}$ & $N_{A-B}^{i o n}$ & $N_{A-B}^{c o v}$ & $W_{A-B}$ \\
\hline Hydrogen & $\mathrm{H}-\mathrm{H}$ & 1.000 & 0.000 & 1.000 & 0.000 & 0.000 & 1.000 & 1.000 \\
\hline Fluorine & $\mathrm{F}-\mathrm{F}$ & 1.317 & 0.119 & 1.317 & 0.119 & 0.119 & 1.317 & 1.087 \\
\hline Hydrogen fluoride & $\mathrm{H}-\mathrm{F}$ & 0.622 & 0.048 & 1.260 & 0.498 & 0.268 & 0.941 & 0.946 \\
\hline Lithium fluoride & $\mathrm{Li}-\mathrm{F}$ & 0.278 & 0.228 & 1.420 & 1.767 & 0.998 & 0.849 & 1.318 \\
\hline Carbon monoxide & $\mathrm{C}-\mathrm{O}$ & 2.554 & 0.431 & 3.834 & 0.925 & 0.678 & 3.196 & 2.916 \\
\hline Carbon dioxide & $\mathrm{C}-\mathrm{O}$ & 3.728 & 0.118 & 3.529 & 1.097 & 0.483 & 2.389 & 2.213 \\
\hline Water & $\mathrm{O}-\mathrm{H}$ & 2.498 & 0.665 & 0.723 & 0.031 & 0.182 & 0.984 & 0.966 \\
\hline Ammonia & $\mathrm{N}-\mathrm{H}$ & 3.656 & 0.362 & 0.806 & 0.019 & 0.070 & 1.007 & 0.982 \\
\hline Methane & $\mathrm{C}-\mathrm{H}$ & 4.394 & 0.062 & 0.886 & 0.009 & 0.012 & 0.984 & 0.989 \\
\hline Ethane & $\mathrm{C}-\mathrm{C}$ & 4.318 & 0.040 & 4.318 & 0.040 & 0.011 & 1.169 & 1.092 \\
\hline Ethane & $\mathrm{C}-\mathrm{H}$ & 4.318 & 0.040 & 0.898 & 0.007 & 0.008 & 0.949 & 0.969 \\
\hline Ethylene & $\mathrm{C}-\mathrm{C}$ & 4.263 & 0.028 & 4.263 & 0.028 & 0.015 & 2.220 & 2.120 \\
\hline Ethylene & $\mathrm{C}-\mathrm{H}$ & 4.263 & 0.028 & 0.891 & 0.008 & 0.007 & 0.918 & 0.952 \\
\hline Acetylene & $\mathrm{C}-\mathrm{C}$ & 4.218 & 0.023 & 4.218 & 0.023 & 0.018 & 3.199 & 3.100 \\
\hline Acetylene & $\mathrm{C}-\mathrm{H}$ & 4.218 & 0.023 & 0.832 & 0.015 & 0.010 & 0.874 & 0.936 \\
\hline Butadiene & $\mathrm{C}_{1}-\mathrm{C}_{2}$ & 4.262 & 0.028 & 4.192 & 0.025 & 0.013 & 2.028 & 1.971 \\
\hline Butadiene & $\mathrm{C}_{1}-\mathrm{C}_{3}$ & 4.262 & 0.028 & 4.192 & 0.025 & 0.000 & 0.037 & 0.036 \\
\hline Butadiene & $\mathrm{C}_{1}-\mathrm{C}_{4}$ & 4.262 & 0.028 & 4.262 & 0.028 & 0.001 & 0.132 & 0.126 \\
\hline Butadiene & $\mathrm{C}_{2}-\mathrm{C}_{3}$ & 4.192 & 0.025 & 4.192 & 0.025 & 0.007 & 1.136 & 1.125 \\
\hline Benzene & $\mathrm{C}_{n}-\mathrm{C}_{n+1}$ & 4.191 & 0.025 & 4.191 & 0.025 & 0.009 & 1.491 & 1.475 \\
\hline Benzene & $\mathrm{C}_{n}-\mathrm{C}_{n+2}$ & 4.191 & 0.025 & 4.189 & 0.026 & 0.000 & 0.037 & 0.036 \\
\hline Benzene & $\mathrm{C}_{n}-\mathrm{C}_{n+3}$ & 4.191 & 0.025 & 4.191 & 0.025 & 0.001 & 0.124 & 0.123 \\
\hline
\end{tabular}

First order density matrix was generated using DFT/B3LYP method and 3-21G basis set

a measure of assymetry of electron density distribution between atoms $\mathrm{A}$ and $\mathrm{B}$, and $N_{A-B}^{c o v}$ (as well as $W_{A-B}$ ) as a measure of atom's interaction through the bond density. In case of $\mathrm{F}_{2}$ molecule IT-covalency index reaches considerably higher value than Wiberg's covalency index, whereas IT-ionicity index assumes low but not negligible value (comparable with $\mathrm{N}-\mathrm{H}$ or $\mathrm{O}-\mathrm{H}$ ). If follows directly from analysis of onecenter IT-valency indices of both $\mathrm{F}$ atoms that slight "ionicity" of chemical bond F-F $\left(N_{A-B}^{i o n} \cong 1.12\right.$ ) does not result from charge transfer (CT) effect but it is associated with lone pairs (LP) influence. It suggests that lone electron pairs of atom have an influence on its chemical bonds with other atoms. This impact depends on a number of LP's and it decreases in the following order: F (3 LPs), O (2 LPs), N (1 LP), C (0 LPs). Therefore, by comparison of $W_{A-B}, N_{A-B}^{c o v}$ and $N_{A-B}^{i o n}$ we can obtain some extra information about molecular system of interest.

\section{Summary}

In this work we have outlined two novel information systems with separated communication channels in which we have studied independently flows of information about 
atomic orbital occupations and-alternatively_flows of information about bonding shares within LCAO MO theory. We have proposed an output-reduction scheme supported by separation of directed flows $A \rightarrow B$ and $B \rightarrow A$ which allows one to define one-center components of atomic IT-valency as well as two-center components of IT-bond order indices. These entropy descriptors comprise an alternative for entropic multiplicities from [10]. We have also demonstrated that $N_{A-B}^{c o v}$ and $N_{A-B}^{i o n}$ successively complements Wiberg's covalency index $W_{A-B}$ providing an extra-information about chemical bond character (polarization). The novel bond descriptors are still in need of thorough examination and comparison with other bond indices such as differential bond orders $[13,14]$ or ionicity indices from purely probabilistic models of chemical bond [15]. These studies are currently in progress.

Open Access This article is distributed under the terms of the Creative Commons Attribution Noncommercial License which permits any noncommercial use, distribution, and reproduction in any medium, provided the original author(s) and source are credited.

\section{References}

1. R.F. Nalewajski, Information Theory of Molecular Systems (Elsevier, Amsterdam, 2006)

2. R.F. Nalewajski, Information Origins of the Chemical Bond (Nova Sc., Hauppauge, 2010)

3. C.E. Shannon, Bell Syst. Tech. J. 27, 379, 623 (1948)

4. R.F. Nalewajski, J. Math. Chem. 43, 265 (2008)

5. R.F. Nalewajski, Mol. Phys. 104, 3339 (2006)

6. R.F. Nalewajski, Int. J. Quantum Chem. 109, 425, 2495 (2009)

7. R.F. Nalewajski, Mol. Phys. 103, 451 (2005)

8. R.F. Nalewajski, J. Math. Chem. 38, 43 (2005)

9. R.F. Nalewajski, Theor. Chem. Acc. 114, 4 (2005)

10. R.F. Nalewajski, D. Szczepanik, J. Mrozek, Adv. Quantum Chem. 61, in press

11. K.A. Wiberg, Tetrahedron 24, 1083 (1968)

12. N. Abramson, Information Theory and Coding (McGraw-Hill, New York, 1963)

13. R.F. Nalewajski, J. Mrozek, J. Quantum Chem. 51, 187 (1994)

14. R.F. Nalewajski, J. Mrozek, G. Mazur, Can. J. Chem. 100, 1121 (1996)

15. A. Mohajeri, P. Dasmeh, Int. J. Mod. Phys. C 18, 1795 (2007) 